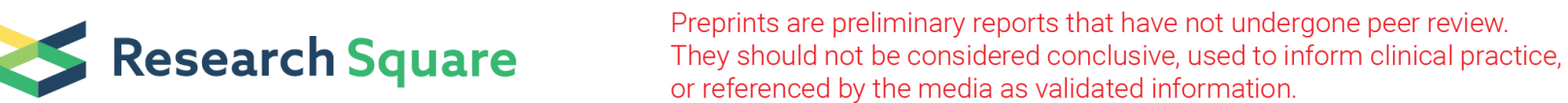

\section{Adaptive evolution of low salinity tolerance and hypoosmotic regulation in a euryhaline teleost, Takifugu obscurus}

\section{Hanyuan Zhang}

Key Laboratory of Aquatic Genomics, Ministry of Agriculture, CAFS Key Laboratory of Aquatic Genomics and Beijing Key Laboratory of Fishery Biotechnology, Chinese Academy of Fishery Sciences, Fengtai, Beijing, China

\section{Jilun Hou}

Beidaihe Central Experiment Station, Chinese Academy of Fishery Sciences, Qinhuangdao, Hebei, China

\section{Haijin Liu}

Breeding Laboratory, Dalian Tianzheng Industry Co. Ltd., Dalian, Liaoning, China

\section{Haoyong Zhu}

Wuxi Fisheries College, Nanjing Agricultural University, Wuxi, China

\section{Gangchun Xu}

Freshwater Fisheries Research Centre of Chinese Academy of Fishery Sciences, Wuxi, China

Jian Xu ( $\nabla$ xuj@cafs.ac.cn )

Chinese Academy of Fishery Sciences https://orcid.org/0000-0003-0274-4268

\section{Research article}

Keywords: Takifugu, low salt-tolerance, hypoosmotic regulation, population genomics, genome resequencing

Posted Date: October 30th, 2019

DOI: https://doi.org/10.21203/rs.2.16620/v1

License: (c) (i) This work is licensed under a Creative Commons Attribution 4.0 International License. Read Full License

Version of Record: A version of this preprint was published at Marine Biology on June 3rd, 2020. See the published version at https://doi.org/10.1007/s00227-020-03705-x. 


\section{Abstract}

Background The mechanism of osmoregulation is crucial for maintaining growth, development, and life activities in teleosts. Takifugu obscurus, the only euryhaline species in the genus Takifugu, is a proper model organism for studying the mechanism of low salt-tolerance and hypoosmotic regulation.

Results In this study, whole genome sequencing data were obtained from 90 pufferfish representing five species within this genus, T. rubripes, T. obscurus, T. flavidus, T. niphobles, and T. bimaculatus. Using a phylogeny, PCA, and population structure analyses, we observed similar amounts of population genetic differentiation among species. The five species are closely related to each other and have differentiated within a relatively short period, while T. bimaculatus and T. flavidus shared the most similar genetic backgrounds. We further identified hundreds of genes under selection related to hypoosmotic regulation between T. obscurus and other Takifugu species, including 16 representative genes involving ion transporters ( atp1a3 , atp2a2, atp2a3 , slc13a1, slc5a8, slc12a2, slc12a4 , slc26a2, scn1b , and kcna $2 / 3 / 10$ ), genes involved in hormone regulation ( fyn , prlr , and grb2 ), and a gene associated with water absorption ( aqp3).

Conclusions: Our findings provide preliminary insight into the mechanism of osmoregulation and will facilitate follow-up validation of candidate genes related to osmoregulation in T. obscurus .

\section{Background}

Pufferfish are a group of migratory benthic teleost fishes belonging to the genus Takifugu (Tetraodontiformes, Tetraodontidae). They are naturally distributed in the Western Pacific Ocean, spanning the Bohai Sea, Yellow Sea, East China Sea, and the Sea of Japan. Some pufferfish, e.g., Takifugu rubripes, Takifugu flavidus, are commercially important fish in China, Japan, and Korea due to their high nutritional value and extraordinary flavor [1]. The genus Takifugu includes 25 species, most of which are closely related, that are able to produce fertile hybrids (e.g., T. rubripes $\times$ T. niphobles, $T$. rubripes $\times$ T. flavidus) [2]. Hybrids can occur in wild populations or in artificially-crossed breeding. The extensive existence of hybrids is mainly due to the relatively recent evolutionary time scale of Takifugu species diversification. Takifugu species underwent explosive speciation in the marine waters of East Asia within 1.8-5.3 Ma during the Pliocene [3]. This was consistent with the theoretical result [4] that more recently diverged species pairs are more likely to produce viable hybrids. It should not go unnoticed that Takifugu species display speciated-related phenotypic variations that could potentially improve our understanding of speciation mechanisms $[5,6]$.

Pufferfish have speciated and radiated under different salinity levels of the aquatic environment. What is noteworthy is that the habitats of most pufferfish are restricted to salt water, with only one exception that can survive and develop normally in freshwater. T. obscurus, as that exceptional case, is a euryhaline fish that can survive in both freshwater and saline water [7]. In its natural habitat, T. obscurus is an anadromous fish that migrates to freshwater to spawn, and newly hatched larvae live there for months 
[8]. Meanwhile, artificial culture of T. obscurus has developed rapidly in China, and the fish are able to grow and reproduce in freshwater over the entire life cycle [9]. Some pufferfish species have the potential to adapt to low salinity to some extent; such species include T. niphobles, which is considered as a peripheral freshwater fish that can survive in freshwater for several days [10]. Fluctuating salinity, an important environmental factor, directly influences the fluid osmotic pressure of fish, in turn impacting the survival, growth, and reproduction of the fish $[11,12]$.

Variation in salinity could lead to a series of physiological responses in osmoregulation-related organs and tissues, including kidney, gill, and intestine $[7,13,14]$. The mechanism of salinity adaptation in teleosts is a complex physiological procedure that cannot be examined using freshwater fish. As an ideal model, the mechanisms of low salinity tolerance differences between T. obscurusand other Takifugu species have been studied from different aspects. In general, T. obscurus has a solid osmotic adjustment capability and a superior osmoregulatory mechanism. In regard to physiological structure, T. obscurus has a freshwater fish-type nephron with a distal tubule in the kidney, while other Takifugu species have a seawater fish-type nephron without the distal tubule [7]. Two cotransporters, $\mathrm{Na}^{+}-\mathrm{K}^{+}-\mathrm{Cl}^{-}$cotransporter 2 (NKCC2) and $\mathrm{Na}^{+}-\mathrm{Cl}^{-}$cotransporter (NCC), have been demonstrated to be differentially expressed in the distal nephrons of T. obscurus and other Takifugu fishes during the process of $\mathrm{NaCl}$ reabsorption [15]. The gill is another important osmoregulatory organ for maintaining ion gradients in teleosts [16]. $\mathrm{Na}^{+} / \mathrm{K}^{+}$ATPase (NKA), as a key enzyme of ion transport maintaining the $\mathrm{Na}^{+}$and $\mathrm{K}^{+}$gradient, is mainly distributed in the chloride cells of the gills and has a crucial function in the ion exchange and osmoregulation in T. obscurus [17]. The activity of NKA changes dramatically with the variation of environmental salinity [18]. In hypoosmotic environments, the intestine plays a pivotal osmoregulatory role in absorbing water and balancing ions through the intestinal epithelia [14]. Meanwhile, kidneys and gills respond to hypoosmotic conditions by participating in the excretion of hypotonic urine and transport of ions. Slc26 has been demonstrated to be an important gene family for exchange of anions such as $\mathrm{HCO}_{3}{ }^{-}$and $\mathrm{SO}_{2}{ }^{-}$[19]. $\mathrm{HCO}_{3}{ }^{-}$and $\mathrm{SO}_{2}{ }^{-}$transporters, which are mainly located in the intestinal epithelial cells and the proximal tubules of the kidney, respectively, are two genes closely related to the adaptation to a seawater environment in T. obscurus [20,21].

In brief, the mechanism of osmoregulation plays a vital role in maintaining life activities in teleosts and has been studied from various aspects at the organ and tissue levels as well as at the cellular and ion channel protein levels. However, the specific genes and co-regulators within their upstream and downstream pathways regulating body fluid osmotic pressure in teleostshave not been well characterized. Therefore, T. obscurus could serve as an ideal experimental model for investigating the molecular mechanisms of osmoregulation and adaptation to low-salinity environments in teleosts. Considering that the complete genome sequence of $T$. obscurus is not yet available, given the high genomic similarity among Takifugu species, the genome of its closely-related species $T$. rubripes could be considered as an alternative. T. rubripes was one of the earliest model species for vertebrates because of its compact genome size ( $400 \mathrm{Mb})$ and genetic repertoire in comparison to other fishes [22]. A draft sequence of the pufferfish was generated using a whole-genome shotgun strategy for T. rubripes [23]. A 
comprehensive genetic map of $T$. rubripes [24] has been generated using 1220 microsatellites that facilitated anchoring the assembled scaffolds to the 22 chromosomes of $T$. rubripeson the basis of preliminary research [25]. This consolidated genome map covered $86 \%$ of the genome assembly, and $72 \%$ of the scaffolds have been oriented by integrating with the genetic linkage map (http://www.fugusg.org/). This genome has been widely used as a valuable resource for comparative genomic and subsequent genetic analysis [26-28]. Furthermore, high-throughput sequencing has been developing rapidly during the last decade. Whole genome re-sequencing is already widely used as a routine tool for identifying genomic differences of samples and for detecting genetic variation.

In this study, the whole genomes of five closely-related pufferfish species were sequenced using Illumina HiSeq X Ten platform. Using the high-quality genomic sequencing data, we elaborated the population genetic differences among the five species. Then, a selective sweep analysis using salt water versus freshwater species was performed to explore the genetic architecture underlying the adaptive evolution of osmoregulation. This study may assist in delineating the genetic diversity of pufferfish and in identifying candidate genes and pathways involved in the regulation networks of low salinity tolerance.

\section{Results}

\section{Sample collection and genotyping}

A total of five Takifugu populations comprising 90 individuals were collected from three sites in China (Figure 1, Supplementary Table 1).. After quality control of extracted DNA, all of the individuals were resequenced to explore the population structure and the selective signals under adaptive evolution. In total, $438.67 \mathrm{~Gb}$ of sequencing data were generated, while for each individual, $4.87 \mathrm{~Gb}$ sequences with an average of $\sim 12.43$-fold depth per sample were obtained. From $2.92 \mathrm{G}$ raw reads, $2.57 \mathrm{G}$ reads were mapped to the reference genome for SNP calling with an average mapping ratio of $87.87 \%$. After the sequence alignment and filtering, a total of $11,344,115$ candidate SNPs were identified for further analysis (Supplementary Table 2)..

\section{Population structure}

To investigate the genetic relationships of different Takifugu species, a phylogenetic tree was constructed using all of the informative SNPs (Figure 2A).. Each population formed a distinct clade that reflected the evolutionary differences among species as expected. Similar results were observed in the plot of the PCA (principle components analysis) analysis. Overall, the populations of T. rubripes, T. niphobles, and $T$. obscurus clustered separately and tightly, while the individuals of $T$. flavidus and $T$. bimaculatus were closely clustered together and overlapped. This was consistent with the results of phylogenetic analysis in that the clades of T. flavidus and T. bimaculatus shared one main branch point and were adjacent to each other. Four clusters from the PCA were far apart from each other with no confusing admixture. Population structure of the five species was evaluated using the Bayesian clustering program STRUCTURE. As the model $\mathrm{K}=4$ obtained the highest log likelihood value, the population structure for $\mathrm{K}$ $=4$ is shown in Figure 2C. Unsurprisingly, similar results were observed; the five populations were 
separated into three subgroups. Outside the T. rubripes and T. obscurus subgroups, T. flavidus, $T$. niphobles, and T. bimaculatus clustered and shared a common ancestry. T. rubripes, T. niphobles,and T. obscurus showed a simplex inheritance background, while T. flavidus and T. bimaculatus reflected a more admixed genetic structure. T. flavidus and T. bimaculatus have a very similar genetic structure and shared some ancestral sequences with $T$. obscurus. When $\mathrm{K}=2,3$, or 5 , only two subgroups existed, $T$. rubripes and the others (Figure S1). This information, which was consistent with the phylogenetic analysis, also reflected the relatively earlier differentiation time of $T$. rubripes and the close genetic relationships and relatively recent species differentiation of the other four species.

\section{LD decay and pattern of haplotype blocks}

LD (Linkage Disequilibrium) decay was investigated for five populations, and $\mathrm{R}^{2}$ values were calculated by PLINK and sorted by distance ranges. T. flavidus showed the highest $\mathrm{R}^{2}$ value, while the other four populations obtained significantly lower $\mathrm{R}^{2}$ values (Figure $3 \mathrm{~A}$ ). When the distance was greater than $1 \mathrm{~kb}$, the mean $\mathrm{R}^{2}$ values were 0.55 in T. flavidus, $0.40-0.45$ in T. rubripes, T. niphobles, and T. obscurus, and 0.38 in T. bimaculatus (Supplementary Table 3). LD decay of whole samples and saline samples showed higher $\mathrm{R}^{2}$ values than most single populations except for $T$. flavidus. Because of the relatively small sample sizes for $T$. flavidus $(N=8)$ and $T$. bimaculatus $(\mathrm{N}=8)$, haplotypes were constructed only for the populations of T. rubripes, T. niphobles, and T. obscurus using the PLINK "-block" parameter. The distribution of haplotype block lengths was calculated using the R software ggplot2 package (Figure 3B). The block patterns were similar in three populations, while all samples and saline samples showed higher densities in smaller blocks. The maximum block lengths were $146.67 \mathrm{~kb}, 36.51 \mathrm{~kb}$, and $34.22 \mathrm{~kb}$ in $T$. rubripes, T. niphobles, and T. obscurus,respectively, while the corresponding mean block sizes were 3.81 $\mathrm{kb}, 2.17 \mathrm{~kb}$, and $2.42 \mathrm{~kb}$ (Supplementary Table 4)..

\section{Genome-wide selective sweep analysis}

The genetic diversity in certain regions of the genome might be dramatically decreased as a result of natural selection or domestication. Among the five species in this study, the T. obscurus population (Ob group) is more strongly euryhaline compared with the other four species (the Saline group), which is probably due to its unique genetic background. To identify genome regions under selection in the $T$. obscurus genome, we scanned the genome-wide variation and allele frequency spectra based on the approximately 13.69 million SNPs. The $\pi$ ratios ( $\left.\pi_{\text {Saline/Obscurus }}\right)$ were calculated using a $100 \mathrm{~kb}$ slidingwindow approach with $10 \mathrm{~kb}$ steps. In comparison to the Saline group, the Ob group had a significantly lower level of diversity (median $\pi_{\text {Saline/Obscurus }}=2.860$ ), reflecting the fewer recombination events in the Ob population. We identified a total of 1,548 significant windows (top $5 \%$, empirical $\pi$ ratios $\geq 5.856$ ) with median $\pi_{\text {Saline/Obscurus }}=8.230$ that included 554 candidate genes (Supplementary Table 5) based on the $\pi$ ratio analysis. To further validate the genome regions under strong selective pressure in the $\mathrm{Ob}$ population, the genome regions with Fst greater than 0.232 (top 5\%) were also identified, corresponding to 497 candidate genes (Supplementary Table 5).. A total of 379 candidate genes shared by both the $\pi$ ratio and Fst analyses were recognized as potential genes under selection (Figure 4A).. Among these 
selected genes, many are involved in ion transportation and ATPase related pathways; those that have been reported in previous studies include atp1a3 (sodium/potassium-transporting ATPase), s/c13a1 (sodium/sulfate symporter), kcna2/3/10 (potassium voltage-gated channel subfamily A member $2 / 3 / 10$ ), s/c5a8 (sodium-coupled monocarboxylate transporter), and scnb1 (sodium channel subunit beta-1) (Figure 4B).. The diversity in the neighboring regions of these selected genes showed significant decrease in the Ob population compared with the Saline group, indicating possible selective sweeps adapting to the long-term changing osmotic environment. For example, in the kcna gene family, kcna2, $k c n a 3$, and $k c n a 10$ in the same sweep region $(>500 \mathrm{~kb})$ were identified, while another gene, s/c5a8, was also found in this region. To better understand these candidate genes and their potential functions, further GO (Gene Ontology) and KEGG (Kyoto Encyclopedia of Genes and Genomes) enrichment analyses also identified several key genes related to osmotic regulation. Two genes, fyn and atp2a3, were enriched in the GO term "ATP binding," and grb2 was enriched in the "Gap junction" pathway (Figure 4B, Figure S2, and Supplementary Table 6).. The results based on Tajima's D analysis also provided supporting evidence identifying strong selective sweep signals (Figure 5).. The results suggested that the genomes of the $\mathrm{Ob}$ population have significantly evolved adaptations to the euryhaline environment.

As the Saline group contains four species, the genetic divergence among species could well reduce the common background noise when used as a whole. The comparison would then be focused on the species' shared lower osmoregulation ability in contrast to the Ob population. Still, it might be useful to identify more potential genes under selection by one-to-one comparisons between the Ob population and the other four populations (shown as $\mathrm{Bi}, \mathrm{Fl}, \mathrm{Ni}, \mathrm{Ru}$ ). The $\pi$ ratios and Fst values for the four comparisons are shown in Figure 6A, and genes within selected regions are summarized in Supplementary Tables 710. The most abundant selected genes ( 211 genes) were found in the comparison FI vs Ob, while 41 genes, 132 genes, and 92 genes were identified in $\mathrm{Bi} v s \mathrm{Ob}, \mathrm{Ni} v s \mathrm{Ob}$, and $\mathrm{Ru} v s \mathrm{Ob}$, respectively. Despite the result that similar genes were found in all four comparisons of Saline vs Ob (e.g., atp1a3, atp2a3, fyn, and scnb1),, other important ion transporter genes were also identified (Figure 6B),, including s/c12a4 (potassium/chloride transporter) from the Bi vs Ob group and s/c12a2 (sodium/potassium/chloride transporter) and s/c26a2 (anion exchanger) from the Ru vs Ob group, indicating their vital roles in regulating osmotic balance. Additionally, atp2b2 (calcium-transporting ATPase 2) and aqp3 (aquaporin 3) were also discovered in selective sweep regions from three comparisons ( $\mathrm{Bi} v s \mathrm{Ob}, \mathrm{Fl} v s \mathrm{Ob}$, and $\mathrm{Ru} v s$ $\mathrm{Ob}$ ). The prlr (prolactin receptor), which was reported to be involved in salinity tolerance of Nile tilapia [29], was also identified in $\mathrm{Bi}$ vs Ob and FI vs Ob comparisons. GO and KEGG enrichment analyses identified crucial pathways including "neuromast development" (contains s/c26a2)," "integral component of plasma membrane" (contains aqp3),, and "neuroactive ligand-receptor interaction" (contains prlr)," suggesting involvement of core functions of the neural system in the regulation of salinity (Supplementary Table 11).. Further analyses based on Tajima's D differences also provided evidence for previous selective sweep signals (Figure S3).. These results suggested that in pairwise comparisons between the Ob population and the other four populations, the genome of the Ob population displays significant selection signatures indicating enhancement of the species' euryhaline ability. 
The most important genes under selection are summarized in Supplementary Table 12. Ion transporters comprise a large proportion of the candidate genes, while growth and neural development-related genes might play upstream roles in the regulation networks.

\section{Discussion}

Previous phylogenetic analysis of 15 Takifugu species [3] indicated that the main lineage has undergone explosive speciation within 2.4-4.7 Ma, meaning that all species are closely related. From the combined results of the phylogeny, PCA and population structure analysis in our study, four main clades were identified: T. rubripes, T. obscurus, T. niphoblesand the group of T. bimaculatus and T. flavidus. This result is consistent with the ML analysis of 24 Takifugu species using cyt $b, 12 \mathrm{~s}$ rRNA gene sequences and mitogenomic data $[3,30]$ showing that $T$. bimaculatus and $T$. flavidus were clustered together in one branch, while T. rubripes, T. obscurus, and T. niphobles were assigned to different branches.

The genes discovered in selective sweep regions included ion transporters, neural regulators, and growthrelated genes, most of which have been previously reported. The T. obscurus population possesses stronger low-salt tolerance than the other four species, and thus the comparisons were made by grouping the four salt-water species together and searching for potential genes under selection in T. obscurus. As various characteristic differences may be revealed by selection analysis, including growth, body shape, body color, and low-salinity tolerance, the genetic divergence within the saline populations could well attenuate the genetic background noise of other traits, allowing us to focus on their common features such as weakened low-salinity tolerance. Similar strategies have been reported in a previous study by Feng Cheng, who identified selective sweeps for leaf-heading trait in the two species Brassica rapa and Brassica oleracea [31]. Chinese cabbage has a unique heading-trait accession in $B$. rapa, while cabbage is the only accession with the heading trait in $B$. oleracea. The non-heading accessions in each species were grouped together to compare with the Chinese cabbage and cabbage. Thus, genetic divergence of other traits among the non-heading accessions would be balanced and would probably not appear in selective sweep analyses. In our study, pairwise comparisons between T. obscurus and the other four species were made as a supplementary analysis to the group comparisons in order to identify additional candidate genes. We identified a number of $s / c$ genes in the Ob population that were embedded in selected regions. These slc genes encode various transmembrane transporters of anions and cations (e.g., s/c13a1, s/c5a8, s/c12a2, s/c12a4, s/c26a2).. The apical membrane $\mathrm{Na}^{+}$-sulfate cotransporter encoded by s/c13a1 mediates sulfate reabsorption across the renal proximal tubule and intestinal epithelia, promoting osmoregulation in low-salinity waters [32,33]. Similarly, a considerable number of genes were identified encoding energy-dependent ATP-binding transporters (atp1a3, atp2a2, atp2a3) and voltage-gated ion channels ( $\operatorname{scn} 1 b, k c n a 2 / 3 / 10)$.. Among these genes, atp1a3 that codes $\mathrm{Na}^{+} / \mathrm{K}^{+}$-ATPase plays a key role in the $\mathrm{Na}^{+}$and $\mathrm{K}^{+}$gradient balance, and this gene has been reported as being differentially expressed in salinity-varying environments in T. obscurus [18]. The voltage-gated ion channel genes ( $s c n 1 b$ and $k c n a$ family) have been discovered by differential gene expression during spawning [34] and selective sweeps adapting freshwater and alkaline waters in Amur Ide [35, 36]. 
Therefore, the genes encoding transcellular ion transporters and channel proteins are reshaped by natural selection from the variable euryhaline environment. Adapting to lower-salinity environments can lead to varied secretion of hormones (e.g., prlr, ghr, igf) that modulate permeability of cells and prevent ion loss and water absorption [37, 38]. The genes fyn and grb2 were embedded in our enriched pathways of "Focal adhesion," while prlrwas enriched in "Neuroactive ligand-receptor interaction." The aqp3 gene was included in the "integral component of plasma membrane" in our selective sweep analysis; this gene has been reported to be involved in regulating water absorption in renal tubules of Atlantic salmon [39]. The main categories of osmoregulation genes have been covered in our study; however, the detailed mechanisms of gene interaction and regulation of gene expression are not yet fully understood. Further experimental evidence in view of expression or methylation would also be necessary in the future to support our potential candidate genes related to osmoregulation in T. obscurus.

\section{Conclusion}

Using whole genome sequencing data, we analyzed the population relationships of five Takifugu species. The selective signatures of the T. obscurus population were screened for effects on low salt-tolerance and hypoosmotic pressure adjustment capacity. Phylogenetic analysis, PCA, and population structure analysis were conducted, and interspecific genetic relationships among five species within the genus Takifugu were established. In addition, a number of genes within selected regions have indicated potential function in osmotic regulation. Among the results, $16 \mathrm{genes}$ were identified as potential candidate genes that could possibly be associated with hypoosmotic pressure regulation in T. obscurus. These results provide an empirical foundation for further insights into the genetic mechanism of osmoregulation in T. obscurus.

\section{Methods}

\section{Sample collection}

A total of 90 samples from five populations were collected, with 29 T. rubripes, 30 T. obscurus, $8 T$. flavidus, 8 T. bimaculatus, and 15 T. niphobles. The samples of T. rubripes, T. flavidus, and T. bimaculatus were from the Tanghai breeding base, Beidaihe Central Experiment Station, Chinese Academy of Fishery Sciences $\left(119^{\circ} 31^{\prime} 32^{\prime \prime} \mathrm{E}, 39^{\circ} 48^{\prime} 58^{\prime \prime} \mathrm{N}\right.$,).A natural population of $T$. niphobles was obtained from the open seas of Bohai Bay located near Beidaihe, Qinhuangdao, Hebei in China $\left(119^{\circ} 32^{\prime} 55^{\prime \prime} \mathrm{E}, 39^{\circ} 48^{\prime} 56^{\prime \prime} \mathrm{N}\right) . T$. obscuruswere sampled from the Zhongyang pufferfish manor in Hai'an, Jiangsu, China $\left(120^{\circ} 53^{\prime} 58^{\prime \prime} \mathrm{E}\right.$, $\left.32^{\circ} 36^{\prime} 44^{\prime \prime} \mathrm{N}\right)$. The salinity of the corresponding water environment was measured using a salinometer. The water salinity for T. obscurus was $6 \mathrm{ppt}$, while the water salinity for the other Takifugu species was equivalent to the natural salinity of seawater, $30 \mathrm{ppt}$.

Whole genome sequencing and SNP discovery 
The collected fish were euthanized in MS222 solution $(30 \mathrm{mg} / \mathrm{L})$ for three minutes before tissue collection. Genomic DNA of 90 fish was extracted from the caudal fin using DNeasy 96 Blood \& Tissue Kits (Qiagen, Shanghai, China) in accordance with the manufacturer's instructions. The integrity of DNA was checked via electrophoresis on $1.0 \%$ agarose gels. Genomic DNA was quantified by Nanodrop-1000 (Thermo Scientific, Wilmington, DE, USA), qualified by the fluorometer Qubit 4.0 (Thermo Scientific) and diluted to $50 \mathrm{ng} / \mu \mathrm{L}$ for sequencing. The total amount of DNA for whole genome sequencing was no less than $2 \mu \mathrm{g}$ per sample. After DNA quality verification, all individuals belonging to $T$. rubripes $(\mathbb{N}=29), T$. obscurus $(\mathbb{N}=30)$, T. flavidus $(\mathbb{N}=8)$, T. bimaculatus $(\mathbb{N}=8)$, and T. niphobles $(\mathbb{N}=15)$ were used for genome re-sequencing. DNA libraries were constructed and barcoded using the DNA Library Prep Reference Guide (Illumina). Sequencing was performed on the Illumina HiSeq X Ten platform with $150 \mathrm{bp}$ paired-end reads. The average sequencing depth was $10 \times$ coverage per sample. The quality of raw reads was assessed using FastQC (http://www.bioinfor-matics.babraham.ac.uk/projects/fastqc/). The sequences were filtered by the following steps: (1) Potential remnants of adapter sequences were removed; (2) low-quality paired reads with higher than $10 \%$ unrecognized bases in a single-end read were eliminated; and (3) low-quality bases with a Phred quality score lower than 20 were trimmed. After filtering, clean sequencing data were used for SNP calling. The sequencing data from each individual were aligned to the genome assembly of T. rubripes FUGU524] using the Burrows-Wheeler Aligner (BWA) [40]. SNP calling was conducted using SAMTOOLS and BCFTOOLS with default parameters [41].

\section{Phylogeny, principle component analysis, and genetic structure}

To understand the evolutionary relationships of pufferfish species, maximum likelihood (ML) analysis was performed to construct a phylogenetic tree using RAxML version 8 [42]. The bootstrap analysis was conducted by the best-scoring topology with 1000 replicates. The iTOL software (http://itol.embl.de/upload.cgi) was used to display the phylogenetic tree. PCA was conducted using PLINK 1.9 [43] and Genome-wide Complex Trait Analysis (GCTA) software [44]. The population structure was investigated by the Bayesian clustering program STRUCTURE 2.3.1 [45] using all of the SNPs, with 2000 iterations. The number of genetic clusters $\mathrm{K}$ was predefined from two to five. The exported structure matrix was visualized by the StructurePlot 2.0 software [46].

\section{LD decay and haplotype construction}

LD decay for five populations, saline populations, and all samples was estimated within a range of $5 \mathrm{~kb}$ using PLINK 1.9 [43]. The average $\mathrm{R}^{2}$ value per $\mathrm{kb}$ region was calculated and plotted against the physical distances of SNPs in units of kb. Haplotype blocks that showed strong linkages were constructed using the PLINK "-blocks" parameter for each population.

\section{Identification of selective signatures between populations}

Whole genome scanning between freshwater species (Ob: T. obscurus, $\mathrm{n}=30$ ) and saline water species (Saline: T. rubripes, T. flavidus, T. niphobles and T. bimaculatus, $\mathrm{n}=60$ ), as well as between T. obscurus and the other four species separately, was conducted using three methods: $\pi$, Fst,and Tajima's D tests. 
The $\pi$ distribution was calculated using a sliding window method in VCFTOOLS [47]. The genome was divided into $100 \mathrm{~kb}$ sliding windows, and the stepwise distance was $10 \mathrm{~kb}$. The $\pi$ ratios of Saline/Ob as well as from $T$. obscurus andthe other fourspecieswere calculated, and the ratios were sorted. Fst and Tajima's D test were conducted using VCFTOOLS "-weir-fst-pop" and "-TajimaD" parameters, respectively. The regions with the highest $5 \% \pi$ ratio and Fst values were identified and considered as candidate regions under selective sweeps. The scatter plots of $\pi$ ratios and Fst values were visualized using the $R$ ggplot2 package (http://cran.r-project.org/package = ggplot2).

\section{Enrichment analysis}

Genes involved in the regions of selective sweeps were chosen for functional annotation. GO and KEGG pathway analyses were performed using DAVID $6.8[48,49]$ between $T$. obscurus and four saline water species, as well as between Saline and Ob.

\section{List Of Abbreviations}

SNP: single nucleotide polymorphism; PCA: principal component analysis; NKCC2: $\mathrm{Na}+-\mathrm{K}+-\mathrm{Cl}-$ cotransporter 2; NCC: Na+-Cl- cotransporter; NKA: Na+/K+-ATPase; LD: linkage disequilibrium; GO: Gene Ontology; KEGG: Kyoto Encyclopedia of Genes and Genomes.

\section{Declarations}

\section{Ethics approval and consent to participate}

This study was carried out in accordance with the recommendations of the care and use of animals for scientific purposes set up by the Animal Care and Use Committee of the Chinese Academy of Fishery Sciences (ACUC-CAFS). The sample collection and protocols were approved by the ACUC-CAFS.

\section{Consent for publication}

Not applicable.

\section{Availability of data and material}

The datasets generated during the current study are available in the NCBI SRA repository (https://www.ncbi.nlm.nih.gov/bioproject/PRJNA522329/).

\section{Competing interests}

The authors declare that they have no competing interests.

Funding 
This work was supported by Central Public-interest Scientific Institution Basal Research Fund, CAFS (No. 2017 B003 and No. 2016HY-JC0301) for genome re-sequencing. The work was supported by the National Infrastructure of Fishery Germplasm Resources of China (No. 2019DKA30470) and the National Natural Science Foundation of China (No. 31502151) for DNA extraction and quality control. The work was supported by the China Agriculture Research System (CARS-47-01) for sample collection.

Author's contributions

JX initiated and coordinated the research project. HZ and JX conducted the analysis, and drafted the manuscript. JH, HL, HZ and GX engaged in sample collection and DNA extraction. All authors read and approved the final manuscript.

Acknowledgments

Not applicable.

\section{References}

1.Song L, Liu B, Xiang J, Qian PY: Molecular phylogeny and species identification of pufferfish of the genus Takifugu (Tetraodontiformes, Tetraodontidae). Mar Biotechnol (NY) 2001, 3(4):398-406.

2. Hosoya S, Kai W, Fujita M, Miyaki K, Suetake H, Suzuki Y, Kikuchi K: The genetic architecture of growth rate in juvenile Takifugu species. Evolution; international journal of organic evolution 2013, 67(2):590598.

3. Yamanoue Y, Miya M, Matsuura K, Miyazawa S, Tsukamoto N, Doi H, Takahashi H, Mabuchi K, Nishida M, Sakai H: Explosive speciation of Takifugu: another use of fugu as a model system for evolutionary biology. Molecular biology and evolution 2009, 26(3):623-629.

4.Bolnick DI, Near TJ: Tempo of hybrid inviability in centrarchid fishes (Teleostei: Centrarchidae). Evolution; international journal of organic evolution 2005, 59(8):1754-1767.

5.Noor MA, Feder JL: Speciation genetics: evolving approaches. Nature reviews Genetics 2006, 7(11):851-861.

6.Seehausen O, Terai Y, Magalhaes IS, Carleton KL, Mrosso HD, Miyagi R, van der Sluijs I, Schneider MV, Maan ME, Tachida H et al: Speciation through sensory drive in cichlid fish. Nature 2008, 455(7213):620626.

7.Kato A, Doi H, Nakada T, Sakai H, Hirose S: Takifugu obscurus is a euryhaline fugu species very close to Takifugu rubripes and suitable for studying osmoregulation. BMC physiology 2005, 5:18.

8. Yan M, Li Z, Xiong B: Preliminary results on osmolality response of pufferfish Takifugu obscurus to sudden salinity change. J Appl Ichthyol 2005, 21(2):156-159. 
9. Yang Z, Chen YF: Induced ovulation using LHRHa in anadromous obscure puffer Takifugu obscurus cultured entirely in freshwater. Fish Physiol Biochem 2003, 29(4):323-326.

10.Kato A, Maeno Y, Hirose S: Brief migration of the grass puffer, Takifugu niphobles, to fresh water from salt water. Ichthyol Res 2010, 57(3):298-304.

11.Shi ZH, Huang XX, Fu RB, Wang HP, Luo HZ, Chen B, Liu MH, Zhang D: Salinity stress on embryos and early larval stages of the pomfret Pampus punctatissimus. Aquaculture 2008, 275(1-4):306-310.

12.Sampaio LA, Freitas LS, Okamoto MH, Louzada LR, Rodrigues RV, Robaldo RB: Effects of salinity on Brazilian flounder Paralichthys orbignyanus from fertilization to juvenile settlement. Aquaculture 2007, 262(2-4):340-346.

13.Perry SF: The chloride cell: structure and function in the gills of freshwater fishes. Annual review of physiology 1997, 59:325-347.

14.Grosell M: Intestinal anion exchange in marine fish osmoregulation. The Journal of experimental biology 2006, 209(Pt 15):2813-2827.

15. Kato A, Muro T, Kimura Y, Li S, Islam Z, Ogoshi M, Doi H, Hirose S: Differential expression of Na+-Clcotransporter and $\mathrm{Na}+-\mathrm{K}+-\mathrm{Cl}$ - cotransporter 2 in the distal nephrons of euryhaline and seawater pufferfishes. American journal of physiology Regulatory, integrative and comparative physiology 2011, 300(2):R284-297.

16.Lee KM, Kaneko T, Aida K: Low-salinity tolerance of juvenile fugu Takifugu rubripes. Fisheries Sci 2005, 71(6):1324-1331.

17.Hwang PP, Lee TH: New insights into fish ion regulation and mitochondrion-rich cells. Comp Biochem Phys A 2007, 148(3):479-497.

18.Li JJ, Wang J, Yang LJ, Chen YF, Yang Z: Changes in plasma osmolality and Na+/K+ ATPase activity of juvenile obscure puffer Takifugu obscurus following salinity challenge. Biochem Syst Ecol 2014, 56:111-117.

19.Mount DB, Romero MF: The SLC26 gene family of multifunctional anion exchangers. Pflug Arch Eur J Phy 2004, 447(5):710-721.

20.Kurita Y, Nakada T, Kato A, Doi H, Mistry AC, Chang MH, Romero MF, Hirose S: Identification of intestinal bicarbonate transporters involved in formation of carbonate precipitates to stimulate water absorption in marine teleost fish. Am J Physiol-Reg I 2008, 294(4):R1402-R1412.

21.Kato A, Chang MH, Kurita Y, Nakada T, Ogoshi M, Nakazato T, Doi H, Hirose S, Romero MF: Identification of renal transporters involved in sulfate excretion in marine teleost fish. Am J Physiol-Reg I 2009, 297(6):R1647-R1659. 
22.Brenner S, Elgar G, Sandford R, Macrae A, Venkatesh B, Aparicio S: Characterization of the pufferfish (Fugu) genome as a compact model vertebrate genome. Nature 1993, 366(6452):265-268.

23.Aparicio S, Chapman J, Stupka E, Putnam N, Chia JM, Dehal P, Christoffels A, Rash S, Hoon S, Smit A et al: Whole-genome shotgun assembly and analysis of the genome of Fugu rubripes. Science 2002, 297(5585):1301-1310.

24.Kai W, Kikuchi K, Tohari S, Chew AK, Tay A, Fujiwara A, Hosoya S, Suetake H, Naruse K, Brenner S et al: Integration of the genetic map and genome assembly of fugu facilitates insights into distinct features of genome evolution in teleosts and mammals. Genome biology and evolution 2011, 3:424-442.

25.Kai W, Kikuchi K, Fujita M, Suetake H, Fujiwara A, Yoshiura Y, Ototake M, Venkatesh B, Miyaki K, Suzuki Y: A genetic linkage map for the tiger pufferfish, Takifugu rubripes. Genetics 2005, 171(1):227-238.

26.Zhang X, Mizukoshi M, Zhang H, Tan E, Igarashi Y, Suzuki Y, Mitsuyama S, Kinoshita S, Saito K, Watabe S et al: Ultrahigh-Density Linkage Map Construction Using Low-Coverage Whole-Genome Sequencing of a Doubled Haploid Population: Case Study of Torafugu (Takifugu rubripes). Genes 2018, 9(3).

27. Hosoya S, Suetake H, Suzuki Y, Kikuchi K: Genetic Basis Underlying Behavioral Correlation Between Fugu Takifugu rubripes and a Closely Related Species, Takifugu niphobles. Behav Genet 2015, 45(5):560-572.

28.leda R, Hosoya S, Tajima S, Atsumi K, Kamiya T, Nozawa A, Aoki Y, Tasumi S, Koyama T, Nakamura O et al: Identification of the sex-determining locus in grass puffer (\&ITTakifugu niphobles\&IT) provides evidence for sex-chromosome turnover in a subset of \&ITTakifugu\&/T species. Plos One 2018, 13(1).

29. Yamaguchi Y, Breves JP, Haws MC, Lerner DT, Grau EG, Seale AP: Acute salinity tolerance and the control of two prolactins and their receptors in the Nile tilapia (Oreochromis niloticus) and Mozambique tilapia (O. mossambicus): A comparative study. Gen Comp Endocr 2018, 257:168-176.

30.Zhang YB, He SP: Investigations into the perplexing interrelationship of the Genus Takifugu Abe, 1949 (Tetraodontiformes, Tetraodontidae). Chinese Sci Bull 2008, 53(2):233-244.

31.Cheng F, Sun RF, Hou XL, Zheng HK, Zhang FL, Zhang YY, Liu B, Liang JL, Zhuang M, Liu YX et al: Subgenome parallel selection is associated with morphotype diversification and convergent crop domestication in Brassica rapa and Brassica oleracea. Nat Genet 2016, 48(10):1218-1224.

32.Markovich D: Na+-sulfate cotransporter SLC13A1. Pflug Arch Eur J Phy 2014, 466(1):131-137.

33.Markovich D, Romano A, Storelli C, Verri T: Functional and structural characterization of the zebrafish Nat-sulfate cotransporter 1 (NaS1) cDNA and gene (slc13a1). Physiol Genomics 2008, 34(3):256-264.

34.Cui J, Xu J, Zhang SH, Wang K, Jiang YL, Mahboob S, Al-Ghanim KA, Xu P: Transcriptional Profiling Reveals Differential Gene Expression of Amur Ide (Leuciscus waleckii) during Spawning Migration. Int J 
Mol Sci 2015, 16(6):13959-13972.

35.Xu J, Li JT, Jiang YL, Peng WZ, Yao ZL, Chen BH, Jiang LK, Feng JY, Ji PF, Liu GM et al: Genomic Basis of Adaptive Evolution: The Survival of Amur Ide (Leuciscus waleckii) in an Extremely Alkaline Environment. Molecular biology and evolution 2017, 34(1):145-159.

36.Xu J, Li Q, Xu LM, Wang SL, Jiang YL, Zhao ZX, Zhang Y, Li JT, Dong CJ, Xu P et al: Gene expression changes leading extreme alkaline tolerance in Amur ide (Leuciscus waleckii) inhabiting soda lake. Bmc Genomics 2013, 14.

37. Sinha AK, Liew HJ, Diricx M, Kumar V, Darras VM, Blust R, De Boeck G: Combined effects of high environmental ammonia, starvation and exercise on hormonal and ion-regulatory response in goldfish (Carassius auratus L.). Aquat Toxicol 2012, 114:153-164.

38.Sakamoto T, McCormick SD: Prolactin and growth hormone in fish osmoregulation. Gen Comp Endocr 2006, 147(1):24-30.

39. Engelund MB, Madsen SS: Tubular localization and expressional dynamics of aquaporins in the kidney of seawater-challenged Atlantic salmon. J Comp Physiol B 2015, 185(2):207-223.

40.Li H, Durbin R: Fast and accurate short read alignment with Burrows-Wheeler transform. Bioinformatics 2009, 25(14):1754-1760.

41.Li H, Handsaker B, Wysoker A, Fennell T, Ruan J, Homer N, Marth G, Abecasis G, Durbin R: The Sequence Alignment/Map format and SAMtools. Bioinformatics 2009, 25(16):2078-2079.

42.Stamatakis A: RAxML version 8: a tool for phylogenetic analysis and post-analysis of large phylogenies. Bioinformatics 2014, 30(9):1312-1313.

43. Chang CC, Chow CC, Tellier LC, Vattikuti S, Purcell SM, Lee JJ: Second-generation PLINK: rising to the challenge of larger and richer datasets. GigaScience 2015, 4:7.

44. Yang J, Lee SH, Goddard ME, Visscher PM: GCTA: a tool for genome-wide complex trait analysis. American journal of human genetics 2011, 88(1):76-82.

45.Falush D, Stephens M, Pritchard JK: Inference of population structure using multilocus genotype data: dominant markers and null alleles. Molecular ecology notes 2007, 7(4):574-578.

46.Ramasamy RK, Ramasamy S, Bindroo BB, Naik VG: STRUCTURE PLOT: a program for drawing elegant STRUCTURE bar plots in user friendly interface. SpringerPlus 2014, 3:431.

47.Danecek P, Auton A, Abecasis G, Albers CA, Banks E, DePristo MA, Handsaker RE, Lunter G, Marth GT, Sherry ST et al: The variant call format and VCFtools. Bioinformatics 2011, 27(15):2156-2158. 
48. Huang DW, Sherman BT, Lempicki RA: Systematic and integrative analysis of large gene lists using DAVID bioinformatics resources. Nat Protoc 2009, 4(1):44-57.

49. Huang da W, Sherman BT, Lempicki RA: Bioinformatics enrichment tools: paths toward the comprehensive functional analysis of large gene lists. Nucleic acids research 2009, 37(1):1-13.

\section{Figures}

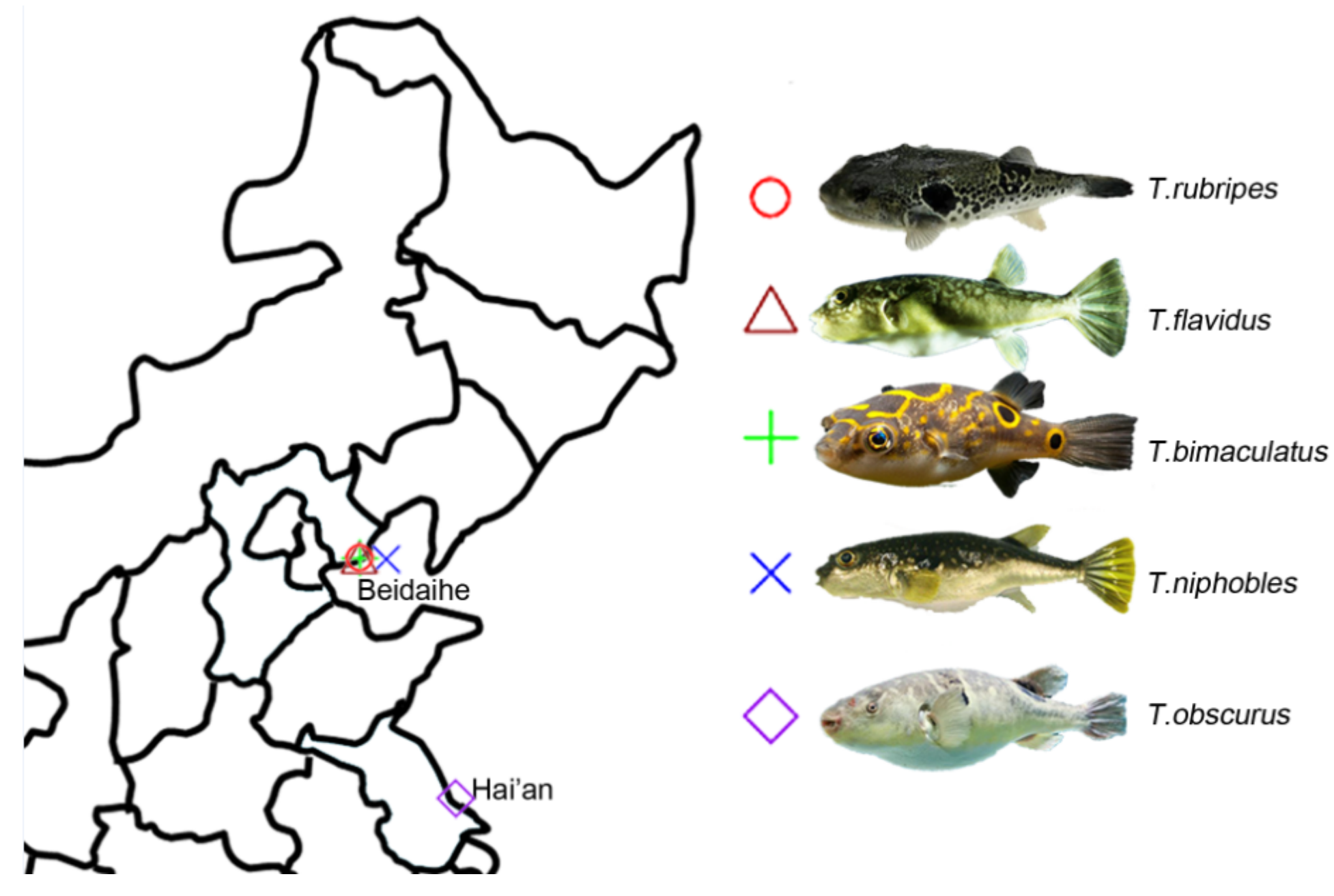

Figure 1

Geographic distribution of five Takifugu populations. Note: The designations employed and the presentation of the material on this map do not imply the expression of any opinion whatsoever on the part of Research Square concerning the legal status of any country, territory, city or area or of its authorities, or concerning the delimitation of its frontiers or boundaries. This map has been provided by the authors. 

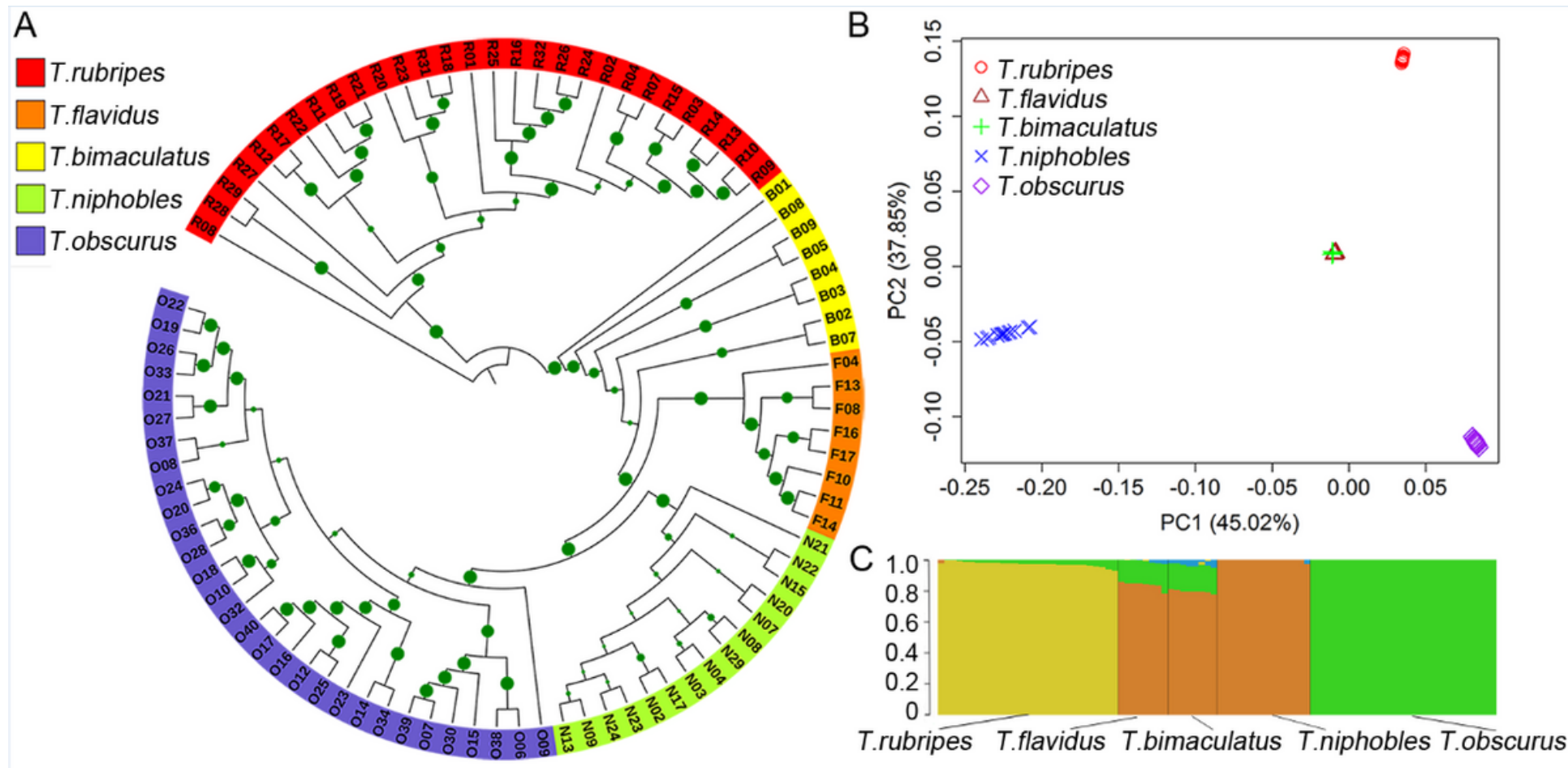

\section{Figure 2}

Phylogeny, PCA, and population structure of five Takifugu populations. (A) The maximum-likelihood phylogenetic tree. The tree was constructed from 11,344,115 SNPs of 90 pufferfish from five populations (red: T. rubripes, orange: T. flavidus, yellow: T. bimaculatus, green: T. niphobles, and purple: T. obscurus). (B) The principal components analysis (PCA) of five Takifugu populations. (C) Genetic structure of the pufferfish inferred by STRUCTURE analysis. The number of ancestral populations $\mathrm{K}=4$ is shown. Each individual has been indicated as a colored segment representing the proportion of its ancestral population. 


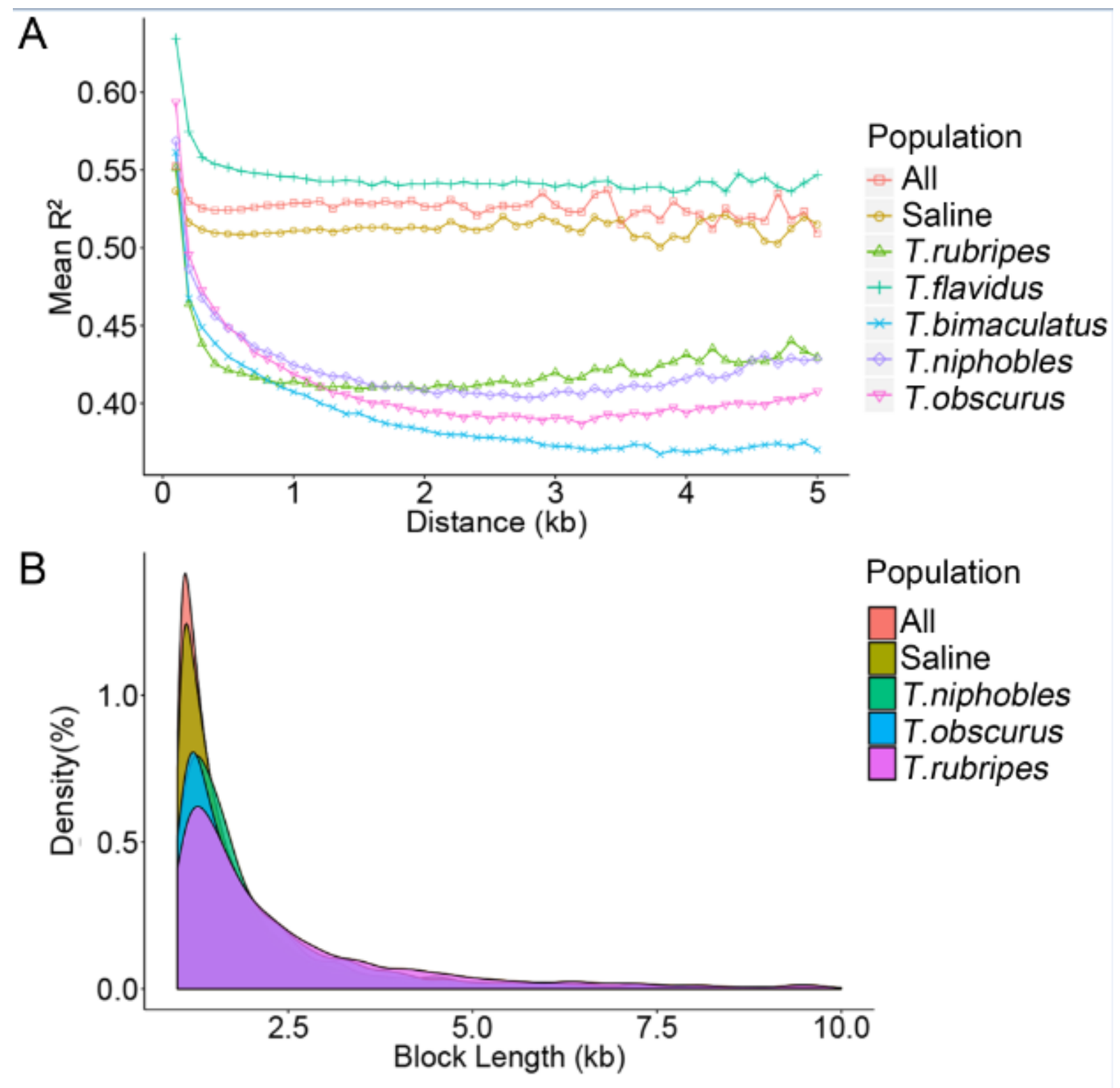

Figure 3

LD decay and haplo-block size distributions. (A) LD patterns of five Takifugu populations, saline populations, and all individuals. (B) Distribution of haplotype block length of T. niphobles, T. obscurus, T. rubripes populations, saline populations, and all individuals. 
A

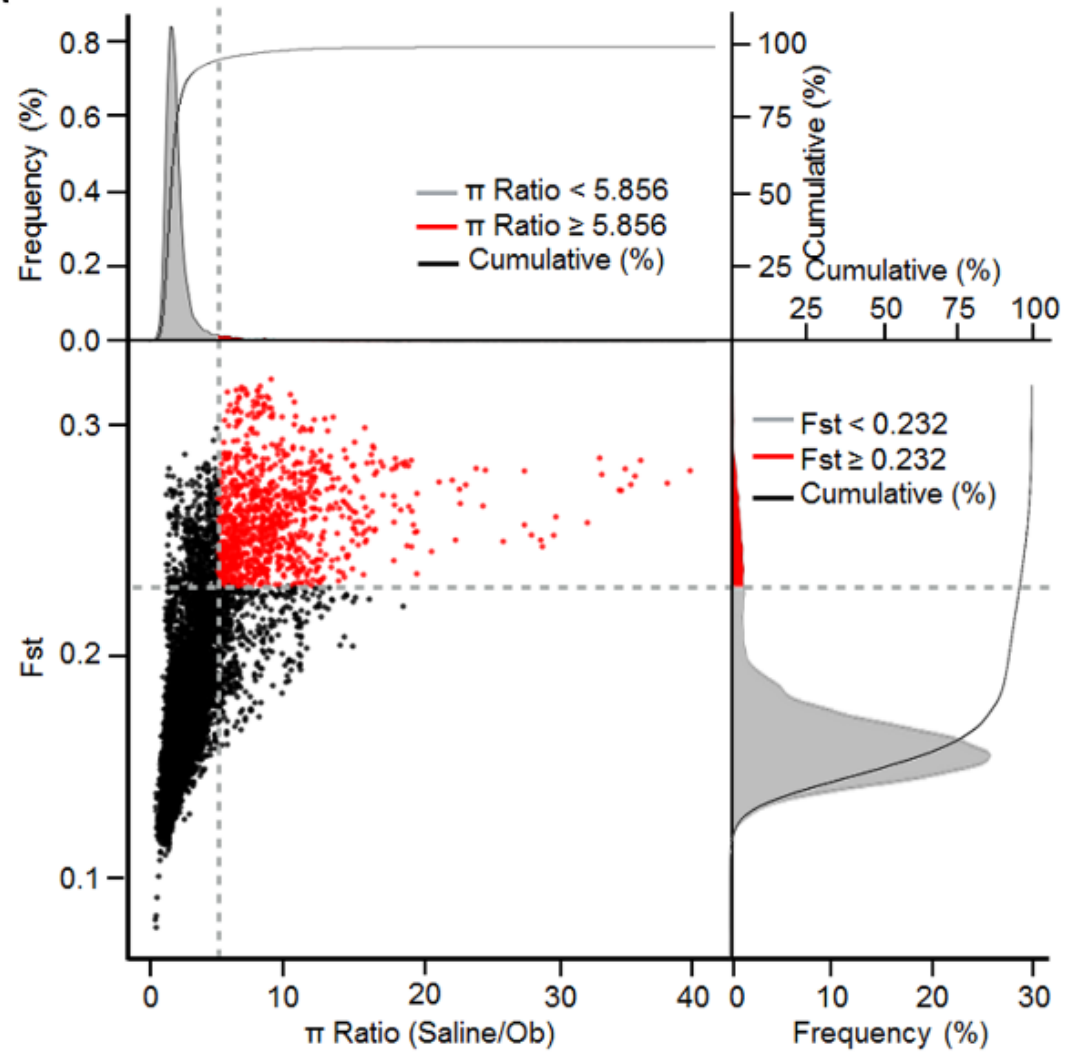

$\mathrm{B}$

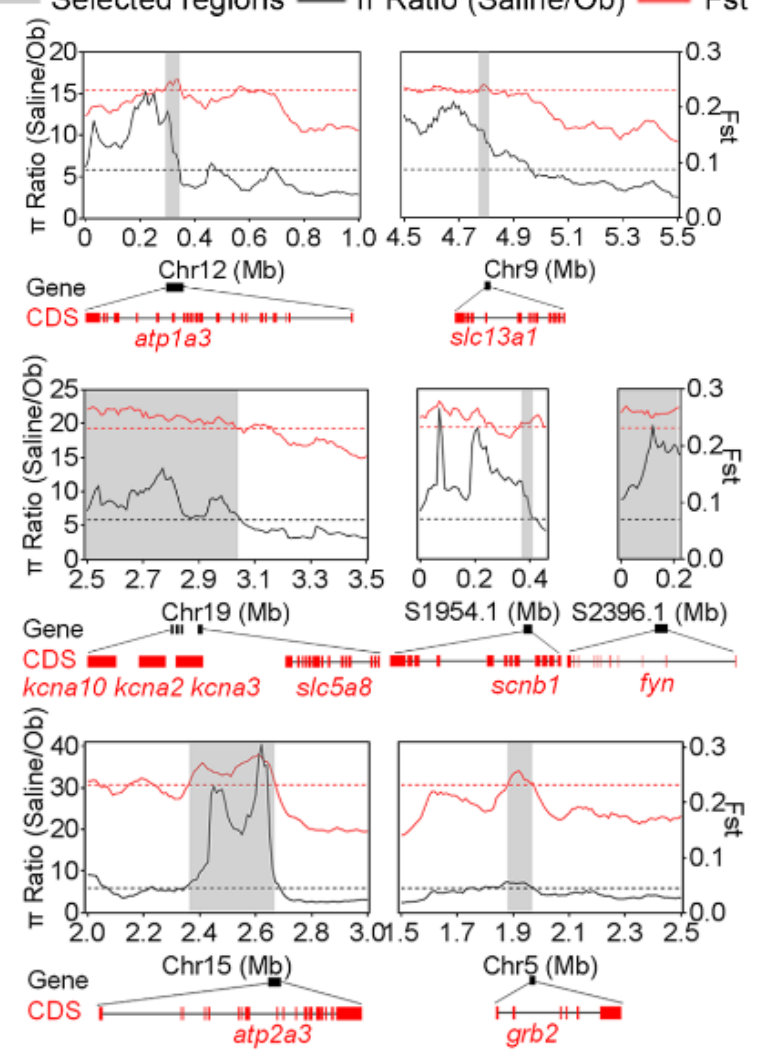

Figure 4

$\mathrm{Pi}$, Fst, and selected genes from selective sweep analysis between the saline populations and $\mathrm{T}$. obscurus. (A) Distribution of $\pi$ ratios and Fst values. Red data points were identified as selective sweeps that passed the thresholds of $\pi$ ratio (the $5 \%$ right tail of the empirical $\pi$ ratio distribution, $\pi$ ratio $\geq 5.856$ ) and Fst (the $5 \%$ right tail of the empirical Fst distribution, Fst $\geq 0.232$ ). (B) Representative genes with strong selective sweep signals in the T. obscurus population. Genome annotations are shown at the bottom, and red bars represent coding sequences. 


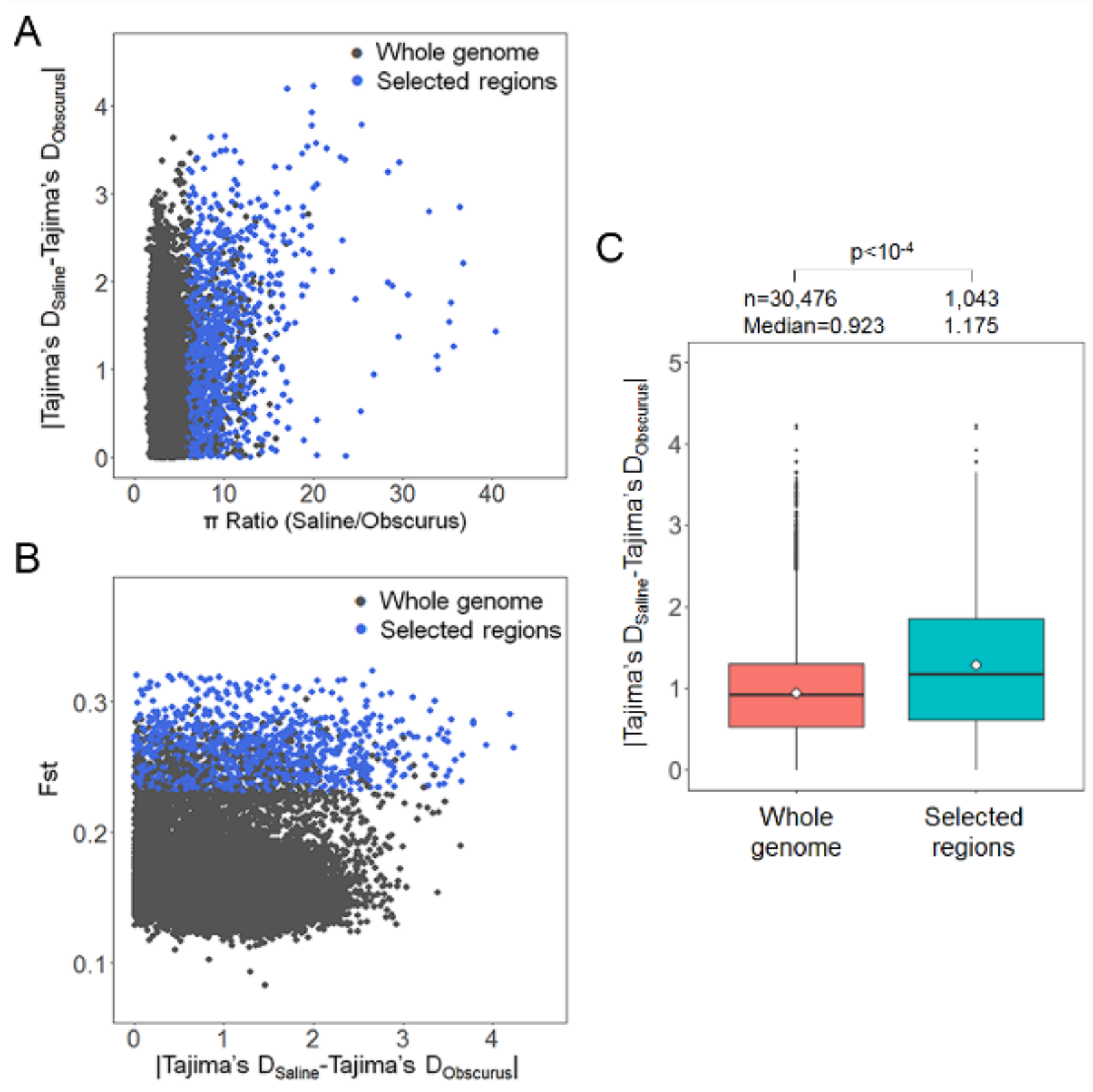

Figure 5

Distribution of $\pi$ ratios, Fst, and Tajima's $D$ values for selective sweep regions between the saline populations and T. obscurus. (A) Distribution of $\pi$ ratios and the absolute differences of Tajima's $D$ values for genomic regions under selective sweeps in comparison with the whole genome. Blue dots denote the selected regions, while black dots denote the unselected regions. (B) Distribution of Fst and the absolute differences of Tajima's $D$ values for genomic regions under selective sweeps in comparison with the whole genome. Blue dots denote the selected regions, while black dots denote the unselected regions. (C) Box plot of the absolute differences of Tajima's D values for genomic regions under selective sweeps in comparison with the whole genome. Boxes denote the values between the 25th and 75th percentiles, and the black transverse line inside the box denotes the median. Black vertical lines denote the values within 1.5 times the quartile values. Outliers are shown as black dots. 

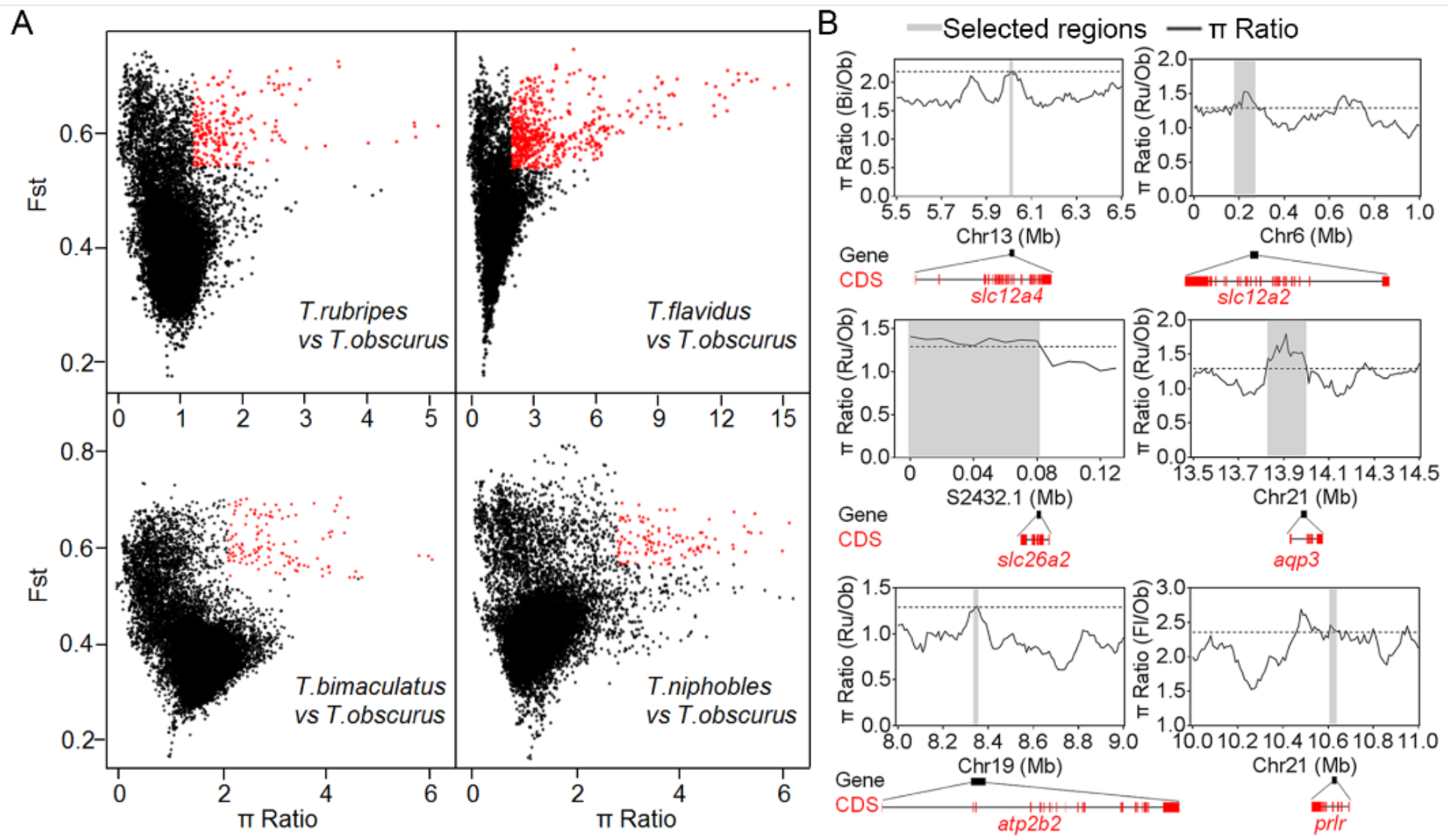

\section{Figure 6}

$\mathrm{Pi}, \mathrm{Fst}$, and candidate genes identified under selective sweep analysis between $\mathrm{T}$. obscurus and four other Takifugu populations. (A) Distribution of $\pi$ ratios and Fst values. Red data points that passed the thresholds of $\pi$ ratio and Fst (the $5 \%$ right tail of the empirical $\pi$ ratio and Fst distributions) located to the upper right were identified as selected regions for the T. rubripes/T. obscurus, T. flavidus/T. obscurus, $T$. bimaculatus/T. obscurus, and T. niphobles/T. obscurus comparisons. (B) Candidate genes with strong selective sweep signals in the $\mathrm{T}$. obscurus population. The $\pi$ ratios were plotted using $10 \mathrm{~kb}$ sliding windows. Genome annotations are shown at the bottom, and red bars represent coding sequences.

\section{Supplementary Files}

This is a list of supplementary files associated with this preprint. Click to download.

- SupplementaryTable112.xlsx

- FigS3.tif

- LegendofSupplementaryMaterials.docx

- Figs2.tif

- NC3RsARRIVEGuidelinesChecklistfillable.pdf

- Figs1.tif 